\title{
Neonatal esophageal trachealization and esophagocarinoplasty in the treatment of flow-limited Floyd II tracheal agenesis
}

\author{
John C. Densmore, MD, ${ }^{\mathrm{a}, \mathrm{b}, \mathrm{c}}$ Keith T. Oldham, MD, ${ }^{\mathrm{a}, \mathrm{b}, \mathrm{c}}$ Kathleen M. Dominguez, MD, ${ }^{\mathrm{d}}$ \\ Elizabeth R. Berdan, MD, ${ }^{\mathrm{a}}$ Michael E. McCormick, MD, ${ }^{\mathrm{e}}$ David J. Beste, MD, ${ }^{\mathrm{e}}$ Louella B. Amos, MD, ${ }^{\mathrm{f}}$ \\ Cecilia A. Lang, RN, MSN, ${ }^{\mathrm{f}}$ Ronald K. Woods, MD, PhD, ${ }^{\mathrm{c}, \mathrm{g}}$ Peter C. Kouretas, MD, PhD, ${ }^{\mathrm{g}}$ and \\ Michael E. Mitchell, MD, ${ }^{\text {a,b,g }}$ Milwaukee and Marshfield, Wis
}

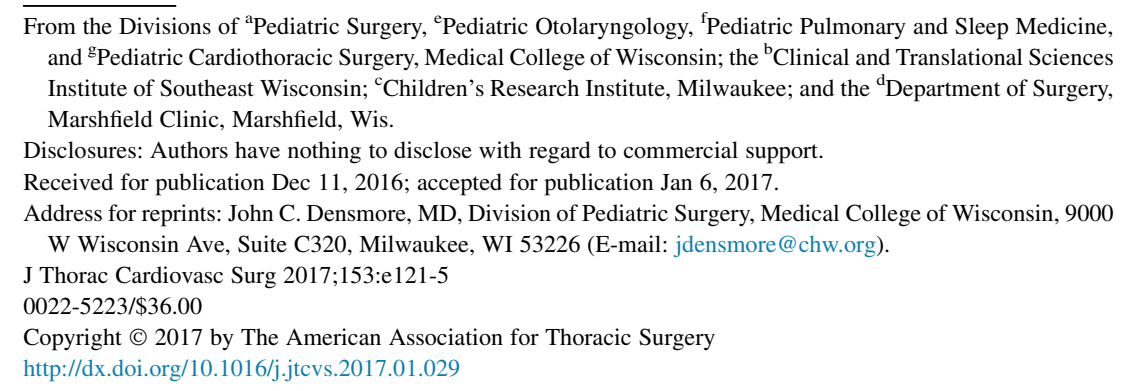

Tracheal agenesis (TA) has been reported to occur in $1 / 50,000$ to $1 / 100,000$ live births. ${ }^{1}$ An exhaustive PubMed review reveals 132 case reports and small series since the condition was first described by Payne ${ }^{2}$ in 1900 . One must assume that numerous postnatal deaths are unreported. Those few patients who survive postnatally require the presence of an adequate tracheoesophageal fistula (TEF) that allows lifesaving esophageal intubation. Surgical intervention with distal esophageal occlusion and gastric decompression then become priorities to allow efficient ventilation to continue. Floyd and colleagues ${ }^{3}$ and Faro and associates ${ }^{4}$ characterized variant patterns of TEF in patients with TA. TA without TEF leads to congenital high airway obstruction syndrome. Four long-term (77-109 months) Japanese survivors of TA have been described by Tazuke and colleagues, ${ }^{5}$ and survival in these cases were achieved by neotrachealization of the esophagus with external stenting and fistulas left in situ. Usui and coworkers ${ }^{6}$ reported on a survivor for whom esophagocarinoplasty was used with external stenting for a flow-limiting fistula. The single previously reported postnatal survivor of TA in the United States, reported by Fonkalsrud and associates ${ }^{7}$ in 1963, died at 6 weeks of age.

The current embryologic pathogenesis for this rare condition remains uncertain. VACTERL (vertebral defects, anal atresia, cardiac defects, tracheoesophageal fistula, renal anomalies, and limb abnormalities) is a known associated sequence that has remained genetically ill-defined. ${ }^{8-10}$ Multiple genomic deletions have been described in affected neonates. Domyan and colleagues ${ }^{11}$ have described a mouse model of tracheal agenesis created by inactivating bone morphogenetic protein receptors (Bmp-1a/b) in the ventral ectoderm. In this model, ectopic respiratory budding occurs with tracheal agenesis. In the mouse, Bmp-4, T-box

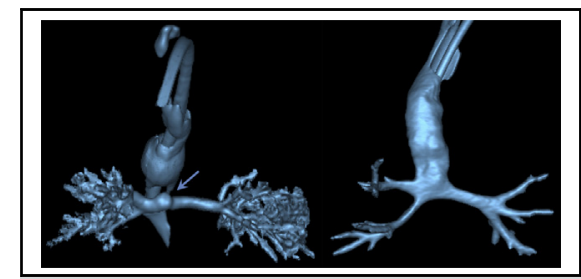

Floyd II tracheal agenesis with fistula (arrow) and after esophagocarinoplasty (right).

Central Message

Tracheal agenesis survival requires regional relationships, an innovative multidisciplinary approach, and family-centered care.

See Editorial Commentary page e127.

transcription factor $(\mathrm{Tbx} 4 / 5)$, Crossveinless2 (CV2), mitogen-activated protein kinase kinase (Mek1/2), and NK2 homeobox (Nkx2.1) receptor knockout pathways comprise the most relevant current models for TA study. ${ }^{12,13}$ Other targets include Forkhead transcription factor (Foxf1), Gli family zinc finger 2 (Gli2/3), Sonic Hedgehog (Shh), retinoic acid receptors $(\operatorname{RAR} \alpha / \beta)$, Smad, Wnt, Notch, Jagged, transforming growth factor- $\beta$, and fibroblast growth factor-all implicated in inadequate foregut separation during organogenesis in the more common esophageal atresia or tracheoesophageal fistula (1/4000 live births). ${ }^{12}$

The child described here is the oldest reported survivor of TA in the United States. The details of the case illustrate the strength of optimized and regionalized children's surgical support, the innovation of multidisciplinary teams, and the role of measured and family-centered intervention when encountering a rare and highly mortal condition. With these tenets in place in an appropriately resourced system, new outcomes become obtainable.

\section{CLINICAL SUMMARY}

A $3.16-\mathrm{kg}$ male infant was born at 37 weeks and 3 days of gestation to a 22-year-old G2P1001 mother by induced vaginal delivery at the Marshfield Clinic (Marshfield, Wis). Polyhydramnios had been identified at 33 weeks' gestation. Neonatologists from the level III neonatal 
intensive care unit at Marshfield Clinic completed resuscitation and attempted endotracheal intubation for respiratory distress. After multiple attempts, the baby was intentionally ventilated through the esophagus, with correction of hypoxia after an estimated 30 minutes. To establish a durable airway, a tracheostomy was attempted but aborted when no trachea could be identified. At this point, the diagnosis of proximal tracheal agenesis with tracheoesophageal fistula led the differential diagnosis. An on-site pediatric surgeon was consulted for progressive abdominal distention, an impending threat to tenuous ventilation. After phone consultation with pediatric surgical teams at a regional level I children's surgery center (Children's Hospital of Wisconsin, Milwaukee, Wis), a laparotomy was performed, the intra-abdominal esophagus was occluded with a large hemoclip, and a Stamm gastrostomy was placed. As a realtime monitor of esophageal occlusion, the gastrostomy tube was submerged in a saline solution trap. The abdomen was temporarily patched open, and the patient was transferred for definitive management.

On arrival to Children's Hospital of Wisconsin on day of life (DOL) 1, cranial ultrasound, renal ultrasound, babygram, echocardiogram, and computed angiogram of the chest were completed. These demonstrated Floyd II tracheal agenesis with a 1-mm diameter tracheoesophageal fistula connecting carina to mid esophagus. In addition, rib fusion anomalies, mild aortic isthmus hypoplasia, and a patent ductus arteriosus with a large left-to-right shunt were identified. No major defects supported concomitant VACTERL sequence. The case was reviewed in a multidisciplinary tracheal team case conference attended by pediatric surgeons, pediatric otorhinolaryngologists, pediatric cardiothoracic surgeons, and the patient's parents. The risks and benefits of surgical intervention as well as a measured stepwise surgical approach were outlined. The major initial concern was the unknown effect of postnatal hypoxia on neurodevelopmental outcome. The simplest durable trachealization of the esophagus was therefore planned with cranial magnetic resonance imaging to detect hypoxic-ischemic changes postoperatively. This delay in imaging was necessary, because magnetic resonance is insensitive to these changes within 7 days of insult.

On DOL 4, a small air leak was detected emanating from the gastrostomy, indicating loosening of the distal esophageal clip. The next day, direct laryngoscopy, patent ductus arteriosus ligation, and an extrapleural approach to esophageal division were undertaken. Laryngoscopic findings were remarkable for identification of a curled epiglottis, arytenoid prominence, diminutive vocal cords, a blindending subglottic airway, and absent laryngeal cleft. The patent ductus arteriosus was clipped through a left anterior approach. The esophagus was approached through a posterolateral 4th interspace right thoracotomy. This exposure allowed identification of the tracheoesophageal fistula and introduction of an adjacent distal EndoGIA 45-mm vascular stapler (Medtronic, Minneapolis, Minn). The esophagus was divided directly adjacent and distal to the insertion of the fistula. Transient hypoxia with mechanical deformation of the fistula demonstrated the fragility of this anatomic situation. The staple line was coated with fibrin glue, and a small chest tube was left adjacent for 48 hours. Finally, the abdomen was entered through the patch, the esophageal hemoclip was removed, and the fascia and skin were primarily closed.

Cranial magnetic resonance imaging completed on DOL 8 demonstrated blood in the occipital horns without hydrocephalus and no parenchymal changes concerning for hypoxic-ischemic brain injury. Weekly surveillance head ultrasounds were continued to monitor for evolution of hydrocephalus. The patient was weaned from narcotics, and gastrostomy feedings were initiated. In expectation of a relatively normal neurodevelopmental prognosis, the infant was returned to the operating room for division of the cervical esophagus into a spit fistula and airway esophagostomy on DOL 12 . The previous cervical midline incision was extended to the sternal notch, the thyroid isthmus divided, and the right recurrent laryngeal nerve was preserved. The operative goal was to place the airway esophagostomy as low as possible to minimize airway dead space. To do this, the esophagus was opened partially and controlled with 3-0 polydioxanone traction sutures. This allowed intubation of the esophagus with a second $3.5-\mathrm{mm}$ cuffed airway tube on the surgical field, exchange of the ventilator circuit, and removal of the original oroesophageal tube. The airway stoma was then matured to the skin edge inferiorly at the level of the sternal notch and the spit fistula matured from the left neck. The length of the new tube measured $6.5 \mathrm{~cm}$ from the neck stoma to tip, and the cuff was inflated. The wound was closed, and the new cervical tube was sutured to the right side of the neck with multiple 3-0 silk sutures affixed to Steri-Strip (3M, St Paul, Minn) mesenteries. One week later (DOL 25), the $3.5-\mathrm{mm}$ cuffed standard tube was exchanged for a $4.0-\mathrm{mm}$ custom Portex Bivona Flextend TTS cuffed tube (Smiths Medical, St Paul, Minn) situated 1 to $1.5 \mathrm{~cm}$ cranial to the visualized tracheoesophageal fistula with a flexible esophagoscope at the bedside.

Cranial ultrasound and computed tomographic scan revealed progressive hydrocephalus requiring intervention on DOL 29. An uncomplicated ventriculoperitoneal shunt was placed. The patient had no neurologic deficits postoperatively and continued to grow on full gastrostomy feedings and mechanical ventilation for approximately 2 months. Our team's original plan was to pursue external esophageal stenting with tracheoesophageal fistula left in situ after 6 months of age. This plan had been conceived with the aid of 3-dimensional printed models generated with the reconstructed airway data from a computed 
tomographic angiogram obtained on DOL 26. On the basis of these models, external stenting with circumferential 20$\mathrm{mm}$ ringed polytetrafluoroethylene for a distance of $5 \mathrm{~cm}$ would allow optimal stenting. By DOL 75, however, the baby began requiring longer inflation times, showed air trapping on chest radiography, and had development of progressive hypercarbia $\left(\mathrm{PCO}_{2}{ }^{80}-100 \mathrm{~mm} \mathrm{Hg}\right)$ despite increasing minute ventilation. These symptoms were clear indication that the fistula was becoming inadequate for gas exchange as the baby continued to grow. Our team had been informed by surgeons at Osaka University about this likely natural history occurring in most cases of tracheal agenesis. In the Osaka University surgeons' experience, this disproportion between overall somatic growth and a relatively fixed fistula is the leading cause of death, affecting most survivors of TA. The priority of external stenting yielded to providing an adequate carinal connection.

In further tracheal team discussions with the family, the problem of fistula inadequacy was detailed, as were reconstructive options. Discussions of suture line failure, granulation tissue, and hemorrhagic stroke were held openly. With fully informed parental consent, the baby was brought to the operating room on DOL 90 for esophagocarinoplasty and external esophageal stenting. Steps detailing this reconstruction are shown in Figure 1. Through a sternotomy, central cannulation was completed, and cardiopulmonary bypass was initiated with systemic heparinization. Because of the previous extrapleural approach for esophageal division, surgical planes were largely unaltered and allowed prompt identification of the stapled end of esophagus and tracheoesophageal fistula. The distal esophagus containing the fistula was resected. A diamond-shaped opening was created on the cephalad side of the right and left main stem bronchi, incorporating the fistula and carina for a total width of $2 \mathrm{~cm}$. The freed esophagus was mobilized $6 \mathrm{~cm}$ proximally and placed inside a $20-\mathrm{mm}$ ringed polytetrafluoroethylene graft sleeve. Then 5-0 pledgeted polypropylene sutures were used to pex the esophageal conduit to the inside of the sleeve radially in 4 quadrants and spaced at $1 \mathrm{~cm}$. Initial $\mathrm{U}$ stitches engaged the muscular esophagus with pledgets interposed within the polytetrafluoroethylene sleeve and were knotted outside the sleeve. The most distal stitches were left untied until the esophagocarinal anastomosis had been completed. This anastomosis was completed in a running fashion with 7-0 polypropylene suture, with knots exteriorized and the remaining pexing
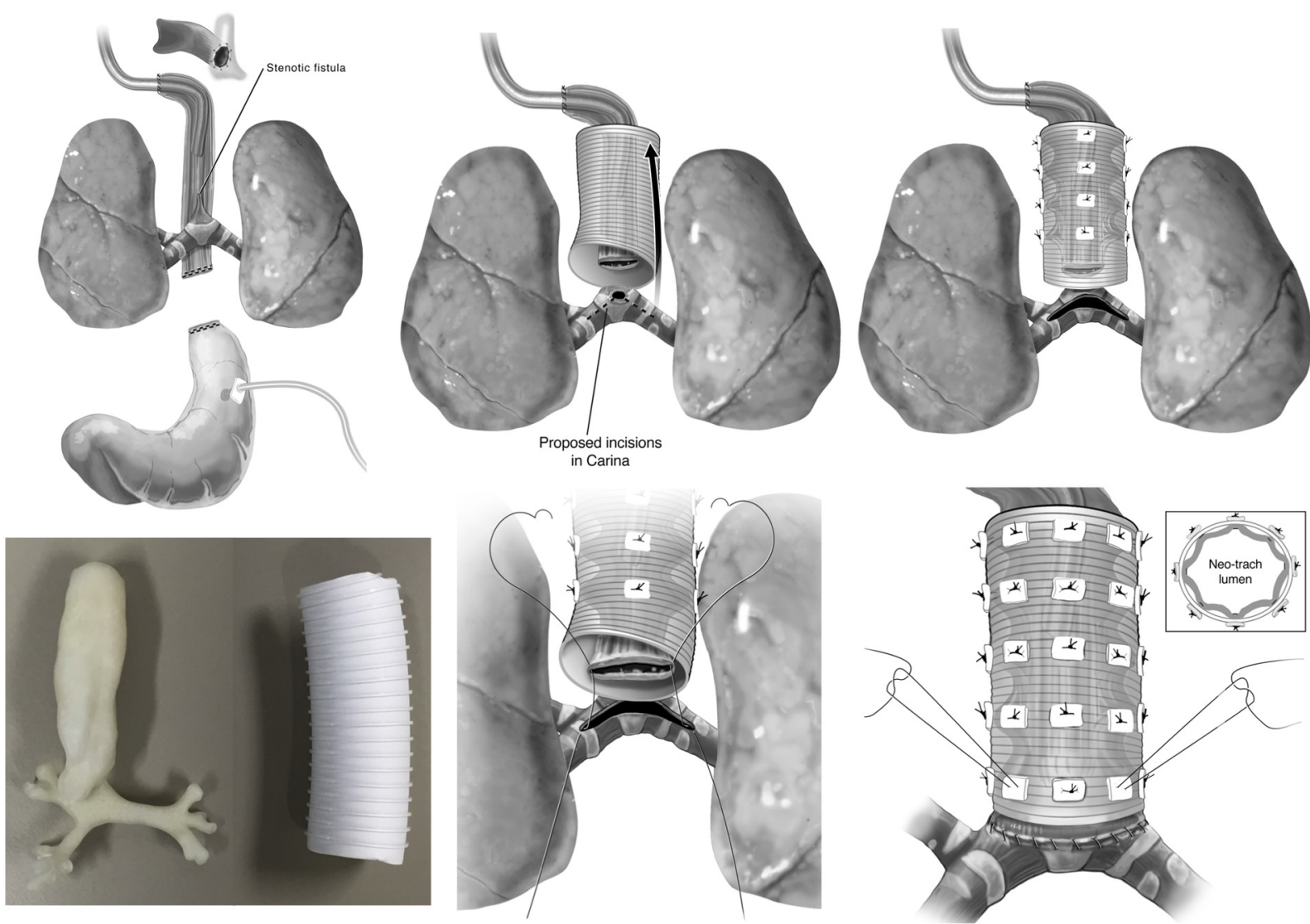

FIGURE 1. Esophagocarinoplasty key steps planned with the aid of a 3-dimensional printed model of the airway including the tracheoesophageal fistula are illustrated. 


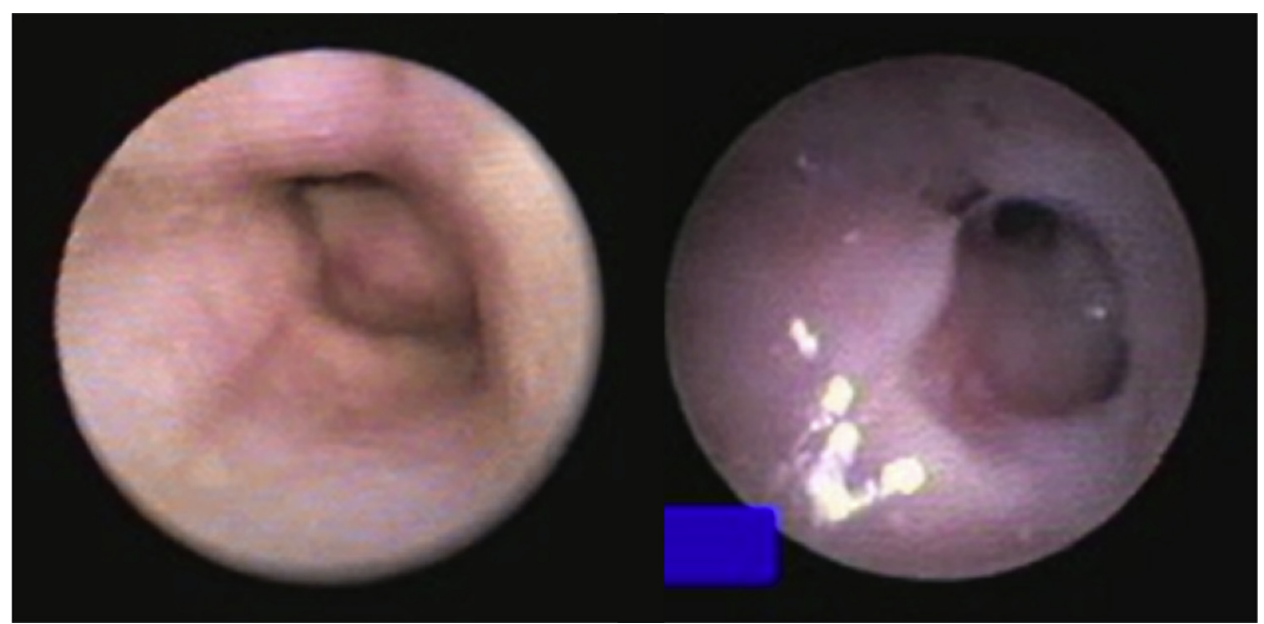

FIGURE 2. Bronchoscopy demonstrating airway patency without evidence of granulation at esophagocarinoplasty obtained on postoperative days 2 (left) and 42 (right).

sutures tied. Intraoperative endoscopy identified areas of mucosal redundancy and obstruction requiring an additional interposed 4 quadrants of pexing sutures. These were placed through fenestrations in the ringed polytetrafluoroethylene sleeve until the airway was patent without positive pressure. The baby was ventilated via the trachealized esophagus, and the suture line was tested with positive pressure $(30 \mathrm{~mm} \mathrm{Hg})$. Bilateral pleural spaces and mediastinum were drained, the infant was decannulated, and the chest was closed. Positive end-expiratory pressure requirements and minute ventilation were notably less postoperatively.

During the subsequent 56 days, the baby was weaned from mechanical ventilation, resting on a ventilator at night for safety. Flexible endoscopy was used to surveil the healing airway and assess the success of external stenting (Figure 2). A heat and moisture exchanger (Thermovent T; Smiths Medical) was attached to the airway to humidify the lungs. Oral skills were maintained with feeds drained through the spit fistula. Repeat bronchoscopy and computed tomographic angiogram of the chest completed on DOLs 115 and 116, respectively, demonstrate a widely patent airway without mechanical ventilation and no granulation tissue. The patient was discharged with the parents on DOL 146 after the parents had mastered all care tasks, including home ventilator for nighttime support, emergency use, and tube exchanges. Ongoing care has been reestablished at the Marshfield Clinic and at the Children's Hospital of Wisconsin.

\section{DISCUSSION}

The history of caring for tracheal agenesis in the current literature paints a picture of emerging innovation and overwhelming mortality. ${ }^{14-16}$ This case illustrates the feasibility of salvage of infants with TA who have adequate an TEF to survive transfer and intervention. All long-term survivors of TA ( $>1$ year of age) have undergone neotrachealization of the esophagus with external stenting as a salvage measure. ${ }^{5,6,17-21}$ A significant difference exists in this case from those survivors, because the concern for neurologic outcome drove a measured surgical response addressing the immediate needs of the infant as they arose. This approach, involving an expert panel of airway specialists, ultimately led to airway reconstruction as described by Usui and coworkers ${ }^{6}$ at the time at which the infant demonstrated the need for further intervention. Success in this case was aided by 3-dimensional modeling obtained through academic relationships, consensus stepwise surgical planning, and inclusive open discussion of possible complications.

The optimal allocation of children's surgical resources has led to the recent review and ongoing verification of hospitals by the American College of Surgeons in a similar manner as College-verified trauma centers. ${ }^{22,23}$ Because TA is a rare, typically lethal, and precipitous condition, prenatally unrecognized TA births are a stress test of any pediatric health care system. As the Children's Hospital of Wisconsin has been recently verified as a level I children's surgery center, initial phone consultation linking this surgical experience to the team at hand in Marshfield proved invaluable to the initial surgical salvage of this infant. In addition, the pediatric surgeon in Marshfield was trained in the Children's Hospital of Wisconsin system. Not surprisingly, previous TA salvages have occurred in smaller countries with nationalized health care systems, namely Japan and South Korea. In these small but dense populations, a similar pattern of care exists naturally.

For rare, lethal, and precipitous conditions such as TA, no center of high volume will ever exist. This case illustrates that the skill sets for the care of infants with TA exist among currently existing high-volume pediatric surgical centers, but this care requires cooperation, innovation, and full 
disclosure of likely outcomes to allow families to be informed and decisive in the care of affected neonates. The paradigm of having cross-discipline problem-focused teams already functioning (eg, tracheal team) aids in the culture of cooperation required to navigate this rare, highly complex, but related condition. In keeping with this principle, a multidisciplinary foregut team will assess timing and feasibility of esophagogastrostomy to achieve oral feedings. Although bioengineered tracheal grafts are currently inadequate, periodic review of translational science may allow the placement of a humidified airway with ciliary function for the patient in the future. This focused and cooperative practice, if translated broadly to pediatric health care systems, will inevitably lead to more survivors. The recent handful of reports of survivors of TA can be offered as indication that surgical treatment is not futile as many recent reports declare. ${ }^{16,24,25}$ When TA is detected prenatally, we believe that delivery at the definitive site of care with an ex utero intrapartum treatment (EXIT) strategy in place would provide the best possible chance for survival and that families may be counseled regarding the welldescribed risks of reconstruction.

\section{References}

1. Manschot HJ, van den Anker JN, Tibboel D. Tracheal agenesis. Anaesthesia. 1994:49:788-90.

2. Payne WA. Congenital absence of the trachea. Brooklyn Med J. 1900;14:568.

3. Floyd J, Campbell DC Jr, Dominy DE. Agenesis of the trachea. Am Rev Respir Dis. 1962;86:557-60.

4. Faro RS, Goodwin CD, Organ CH Jr, Hall RT, Holder TM, Ashcraft KW, et al. Tracheal agenesis. Ann Thorac Surg. 1979;28:295-9.

5. Tazuke Y, Okuyama H, Uehara S, Ueno T, Nara K, Yamanaka H, et al. Long-term outcomes of four patients with tracheal agenesis who underwent airway and esophageal reconstruction. J Pediatr Surg. 2015;50:2009-11.

6. Usui N, Kamiyama M, Tani G, Takama Y, Soh H, Uehara S, et al. Three-stage reconstruction of the airway and alimentary tract in a case of tracheal agenesis. Ann Thorac Surg. 2010;89:2019-22.

7. Fonkalsrud EW, Martelle RR, Maloney JV Jr. Surgical treatment of tracheal agenesis. J Thorac Cardiovasc Surg. 1963;45:520-5.
8. Evans JA, Greenberg CR, Erdile L. Tracheal agenesis revisited: analysis of associated anomalies. Am J Med Genet. 1999;82:415-22.

9. Mandrekar SR, Amoncar S, Pinto RG. Tracheal agenesis with bronchoesophageal fistula in VACTERL/TACRD association. Indian J Hum Genet 2013;19:87-9

10. de Groot-van der Mooren MD, Haak MC, Lakeman P, Cohen-Overbeek TE, van der Voorn JP, Bretschneider JH, et al. Tracheal agenesis: approach towards this severe diagnosis. Case report and review of the literature. Eur J Pediatr. 2012; 171:425-31.

11. Domyan ET, Ferretti E, Throckmorton K, Mishina Y, Nicolis SK, Sun X. Signaling through BMP receptors promotes respiratory identity in the foregut via repression of Sox2. Development. 2011;138:971-81.

12. Sher ZA, Liu KJ. Congenital tracheal defects: embryonic development and animal models. AIMS Genetics. 2016;3:60-73.

13. Li Y, Gordon J, Manley NR. Bmp4 is required for tracheal formation: a nove mouse model for tracheal agenesis. Dev Biol. 2008;322:145-55.

14. Lopez L, Serrano A, Varela A, Freixinet J, Santana R, Beltra R. Tracheal agenesis: an unresolved problem. Thorac Cardiovasc Surg. 1991;39:99-101.

15. Nimbalkar SM, Patel VK, Patel DV, Sethi AR. Tracheal agenesis with tracheooesophageal fistula. J Clin Diagn Res. 2014;8:171-2.

16. Mohammed H, West K, Bewick J, Wickstead M. Tracheal agenesis, a frightening scenario. J Laryngol Otol. 2016;130:314-7.

17. Okuyama H, Kubota A, Kawahara H, Oue T, Tazuke Y. Congenital laryngeal atresia associated with esophageal atresia and tracheoesophageal fistula: a case of long-term survival. J Pediatr Surg. 2006;41:e29-32.

18. Watanabe T, Okuyama H, Kubota A, Kawahara H, Hasegawa T, Ueno T, et al. A case of tracheal agenesis surviving without mechanical ventilation after external esophageal stenting. J Pediatr Surg. 2008;43:1906-8.

19. Fuchimoto Y, Mori M, Takasato F, Tomita H, Yamamoto Y, Shimojima N et al. A long-term survival case of tracheal agenesis: management for tracheoesophageal fistula and esophageal reconstruction. Pediatr Surg Int. 2011;27:103-6.

20. Okuyama H, Sasaki T, Nose S, Saka R, Suzuki M. Jejunal pedicle graft reconstruction of the esophagus in a child with tracheal agenesis. Eur J Pediatr Surg. 2012;22:485-7

21. Park BJ, Kim MS, Yang JH, Jun TG. Tracheal agenesis reconstruction with external esophageal stenting: postoperative results and complications. Korean J Thorac Cardiovasc Surg. 2015;48:439-42.

22. Oldham KT. Optimal resources for children's surgical care. J Pediatr Surg. 2014 49:667-77.

23. Barnhart DC, Oldham KT. Regionalization of children's surgery. Ann Surg. 2016; 263:1067-8

24. Bertholdt C, Perdriolle-Galet E, Bach-Segura P, Morel O. Tracheal agenesis: a challenging prenatal diagnosis-contribution of fetal MRI. Case Rep Obstet Gynecol. 2015;2015:456028.

25. Desai AV, Rao S, Shanbhag PR, Rupani M. Tracheal agenesis: a report of two cases. J Postgrad Med. 2016;62:202-4. 